\title{
Shape Reconstruction of Thin Electromagnetic Inclusions via Boundary Measurements: Level-Set Method Combined with the Topological Derivative
}

\author{
Won-Kwang Park \\ Department of Mathematics, Kookmin University, Seoul 136-702, Republic of Korea \\ Correspondence should be addressed to Won-Kwang Park; parkwk@kookmin.ac.kr
}

Received 15 July 2013; Revised 31 October 2013; Accepted 31 October 2013

Academic Editor: Sotiris Ntouyas

Copyright (c) 2013 Won-Kwang Park. This is an open access article distributed under the Creative Commons Attribution License, which permits unrestricted use, distribution, and reproduction in any medium, provided the original work is properly cited.

An inverse problem for reconstructing arbitrary-shaped thin penetrable electromagnetic inclusions concealed in a homogeneous material is considered in this paper. For this purpose, the level-set evolution method is adopted. The topological derivative concept is incorporated in order to evaluate the evolution speed of the level-set functions. The results of the corresponding numerical simulations with and without noise are presented in this paper.

\section{Introduction}

In general, one of the main purposes of the inverse scattering problem is to determine the characteristics of an unknown target, for example, the shape, location, and internal constitution, based on the measured scattered field data. Therefore, there exist a considerable number of significant inverse scattering problems for identifying the information of arbitraryshaped thin penetrable electromagnetic inhomogeneities or perfectly conducting cracks concealed in structures such as concrete walls of buildings and bridges (refer to [1] and the references therein).

In order to solve related inverse problems, various algorithms have been developed. Among them, an iterative algorithm based on level-set evolution has been established in [2] and many generalizations have been investigated (refer to [3] and the references therein). However, most research studies have focused on the shape reconstruction of volumetric targets. Therefore, in order to reconstruct crack-like targets via level-set evolution, a new concept is required.

In recent work [4], arbitrary-shaped thin penetrable conductivity inclusions have been successfully reconstructed via the level-set method by adopting two different levelset functions. Shape reconstruction of thin electromagnetic inclusions is considered in [5]. However, in [5], only the case of the purely permittivity contrast or permeability contrast with respect to the background material is considered. So, extension to both contrast cases is still remaining as a research topic.

Motivated by the mentioned above, the main objective of this paper is to reconstruct the shape of thin electromagnetic inclusions by adopting the level-set method in the case of both the dielectric permittivity and the magnetic permeability contrasts with respect to the background material. Note that, in general research papers, the evolution speed is evaluated via a complex calculation of the Fréchet derivatives using an adjoint technique. Instead of such evaluations, we incorporate a topological derivative to derive the evolution speed of two level-set functions introduced in [6-10].

This paper is organized as follows. In Section 2, we introduce two-dimensional direct scattering from an arbitraryshaped thin penetrable electromagnetic inclusion and the level-set functions for describing such inclusions. In Section 3 , the evolution speed of the level-set functions is derived via the topological derivative. We present some numerical simulation results in Section 4 to show the effectiveness of the level-set method. Finally, we provide short conclusions in Section 5. 


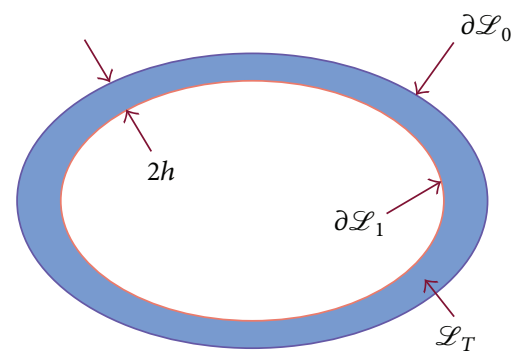

(a)

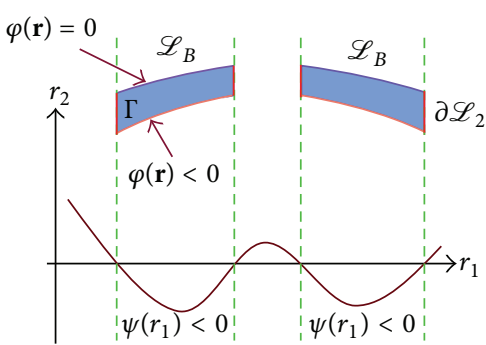

(b)

FIGURE 1: Illustration of the thin inclusion $\Gamma$ using two level-set functions.

\section{Direct Scattering Problem and Two Level-Set Functions: A Brief Survey}

2.1. Two-Dimensional Direct Scattering Problem. Let $\Omega$ be a two-dimensional homogeneous domain with a smooth boundary $\partial \Omega$. Throughout this paper, we assume that $\Omega$ conceals an electromagnetic inclusion $\Gamma$ with thickness $2 h$, which is characterized in the neighborhood of the smooth curve $\sigma$ :

$$
\Gamma:=\{\mathbf{r}+\eta \mathbf{n}(\mathbf{r}): \mathbf{r} \in \sigma, \eta \in(-h, h)\},
$$

where $\mathbf{n}(\mathbf{r})$ is a unit normal to $\sigma$ at $\mathbf{r}$. Throughout this paper, we assume that $\Gamma$ does not touch $\partial \Omega$; that is, we assume that $\Gamma \cap \partial \Omega=\emptyset$.

In this paper, the electric permittivities and magnetic permeabilities of $\Gamma$ and $\Omega$ at a given nonzero frequency are assumed to be known; they are finite valued and they differ (either one or both of them) from the ones of the homogeneous embedding medium. For the sake of simplicity, let $\varepsilon_{0}$ and $\mu_{0}$ denote the permittivity and permeability of $\Omega$, and let $\varepsilon$ and $\mu$ denote those of $\Gamma$, respectively. Then, we can define the following piecewise constants:

$$
\begin{aligned}
& \varepsilon(\mathbf{r})= \begin{cases}\varepsilon_{0} & \text { for } \mathbf{r} \in \Omega \backslash \bar{\Gamma}, \\
\varepsilon & \text { for } \mathbf{r} \in \Gamma,\end{cases} \\
& \mu(\mathbf{r})= \begin{cases}\mu_{0} & \text { for } \mathbf{r} \in \Omega \backslash \bar{\Gamma}, \\
\mu & \text { for } \mathbf{r} \in \Gamma .\end{cases}
\end{aligned}
$$

Let $u_{\Gamma}^{(n)}(\mathbf{r})$ denote a time-harmonic total field that satisfies the following Helmholtz equation:

$$
\nabla \cdot\left(\frac{1}{\mu(\mathbf{r})} \nabla u_{\Gamma}^{(n)}(\mathbf{r})\right)+\omega^{2} \varepsilon(\mathbf{r}) u_{\Gamma}^{(n)}(\mathbf{r})=0 \quad \text { in } \Omega
$$

with boundary condition

$$
\frac{1}{\mu_{0}} \frac{\partial u_{\Gamma}^{(n)}(\mathbf{r})}{\partial \nu(\mathbf{r})}=\frac{1}{\mu_{0}} \frac{\partial \exp \left(i k_{0} \mathbf{d}_{n} \cdot \mathbf{r}\right)}{\partial \nu(\mathbf{r})}=g(\mathbf{r}) \quad \text { on } \partial \Omega
$$

where $k_{0}=\omega \sqrt{\varepsilon_{0} \mu_{0}}, \nu(\mathbf{r})$ denotes the unit outward normal to $\mathbf{r} \in \partial \Omega$, and $\mathbf{d}_{n}$ denotes a vector on the unit circle $\mathbb{S}^{1}$. In the same manner, we denote $u^{(n)}(\mathbf{r})=\exp \left(i k_{0} \mathbf{d}_{n} \cdot \mathbf{r}\right)$ as a background solution of (3) with boundary condition (4).
2.2. Description of Thin Inclusion Using Two Level-Set Functions. Now, we describe the thin inclusion $\Gamma$ by two different level-set functions (see $[4,5]$ for a detailed description). From now on, we assume that the level-set function $\varphi$ is a $\mathscr{C}^{1}$ function. With this assumption, we introduce the zero levelset function $\mathscr{L}_{0}$ and its boundary $\partial \mathscr{L}_{0}$ as

$$
\begin{gathered}
\mathscr{L}_{0}:=\left\{\mathbf{r} \in \mathbb{R}^{2}: \varphi(\mathbf{r}) \leq 0\right\}, \\
\partial \mathscr{L}_{0}:=\left\{\mathbf{r} \in \mathbb{R}^{2}: \varphi(\mathbf{r})=0\right\},
\end{gathered}
$$

respectively. Then, a thin region $\mathscr{L}_{T}$ of thickness $2 h$ and inner boundary $\partial \mathscr{L}_{1}$ can be written as follows:

$$
\begin{gathered}
\mathscr{L}_{T}:=\mathscr{L}_{0} \cap\left\{\mathbf{r}-\eta \mathbf{n}(\mathbf{r}): \mathbf{r} \in \partial \mathscr{L}_{0}, 0 \leq \eta \leq 2 h\right\}, \\
\partial \mathscr{L}_{1}:=\partial \mathscr{L}_{T} \backslash \partial \mathscr{L}_{0},
\end{gathered}
$$

respectively.

In order to describe a thin region of finite length (which is connected or disconnected) in $\Omega$, we assume that another level-set function $\psi$ is also a continuously differentiable function. Let us define a band structure $\mathscr{L}_{B} \subset \mathbb{R}^{2}$ by

$$
\mathscr{L}_{B}:=\left\{\mathbf{r}=\left(r_{1}, r_{2}\right) \in \mathbb{R}^{2}: \psi\left(r_{1}\right)<0\right\} .
$$

Then, we can describe $\Gamma$ as

$$
\Gamma=\left(\mathscr{L}_{T} \cap \mathscr{L}_{B}\right) \cap \Omega
$$

and the boundary $\partial \mathscr{L}_{2}$ of $\Gamma$ for evolving at inclusion tips as

$$
\partial \mathscr{L}_{2}:=\left\{\mathbf{r}=\left(r_{1}, r_{2}\right) \in \partial \Gamma: \psi\left(r_{1}\right)=0\right\} .
$$

Figure 1 shows the illustration of the thin inclusion $\Gamma$ using two level-set functions.

\section{Evolution Speed of the Level-Set Functions and the Topological Derivative}

The evolution of the level-set function $\phi$ satisfies the Hamilton-Jacobi type equation:

$$
\frac{\partial \phi(\mathbf{r})}{\partial t}=\mathbb{F}(\mathbf{r})|\nabla \phi(\mathbf{r})|=0,
$$


where $\phi$ is $\varphi$ or $\psi$ introduced in Section 2.2 and $\mathbb{F}(\mathbf{r})$ is given by (see, e.g., $[4,5])$ the following.

(i) For level-set function $\varphi(\mathbf{r})$ and $\mathbf{r} \in \partial \mathscr{L}_{0}$,

$$
\mathbb{F}(\mathbf{r})=\operatorname{Re}\left(\mathbb{S}(\mathbf{r})\left[\delta\left(\mathbf{r} ; \partial \mathscr{L}_{0}\right)-\delta\left(\mathbf{r}+2 h \mathbf{n}(\mathbf{r}) ; \partial \mathscr{L}_{0}\right)\right]\right) .
$$

(ii) For level-set function $\psi(\mathbf{r})$ and $\mathbf{r}=\left(r_{1}, r_{2}\right) \in \partial \mathscr{L}_{2}$,

$$
\mathbb{F}(\mathbf{r})=\operatorname{Re}\left(\int_{\partial \mathscr{L}_{2}} \mathbb{S}(\mathbf{r}) \delta\left(\mathbf{r} ; \partial \mathscr{L}_{2}\right) \chi(\mathbf{r} ; \mathscr{L}) d r_{2}\right) .
$$

Here, $\mathbb{S}(\mathbf{r})$ denotes the speed function of the level sets that we have to evaluate. In a recent work [5], $\mathbb{S}(\mathbf{r})$ has been derived for the purely permittivity contrast $\left(\varepsilon \neq \varepsilon_{0}\right.$ and $\left.\mu=\mu_{0}\right)$ or permeability contrast $\left(\varepsilon=\varepsilon_{0}\right.$ and $\left.\mu \neq \mu_{0}\right)$ cases by evaluating the Fréchet derivative. In order to evaluate $\mathbb{S}(\mathbf{r})$ for both the permittivity and permeability contrast cases, we introduce the following discrepancy functional:

$$
\begin{aligned}
\mathbb{E}(\Omega) & :=\frac{1}{2} \sum_{n=1}^{N}\left\|u_{\Gamma}^{(n)}\left(\mathbf{r}^{\prime}\right)-u^{(n)}\left(\mathbf{r}^{\prime}\right)\right\|_{L^{2}(\partial \Omega)}^{2} \\
& =\frac{1}{2} \sum_{n=1}^{N} \int_{\partial \Omega}\left(\left|u_{\Gamma}^{(n)}\left(\mathbf{r}^{\prime}\right)-u^{(n)}\left(\mathbf{r}^{\prime}\right)\right|^{2}\right) d S\left(\mathbf{r}^{\prime}\right) .
\end{aligned}
$$

Then, we adopt the following relationship between the evolution speed of level sets and the topological derivative.

Theorem 1. Let $D_{T} \mathbb{E}(\mathbf{r})$ denote the topological derivative of (13); then $D_{T} \mathbb{E}(\mathbf{r})$ satisfies

$$
D_{T} \mathbb{E}(\mathbf{r})= \begin{cases}-\mathbb{S}(\mathbf{r}) & \text { for } \mathbf{r} \in \Gamma \\ \mathbb{S}(\mathbf{r}) & \text { for } \mathbf{r} \in \Omega \backslash \bar{\Gamma} .\end{cases}
$$

Proof. A rigorous derivation can be found in $[6,8,9]$.

Hence, we can evaluate the evolution speed $\mathbb{S}(\mathbf{r})$ by computing the topological derivative $D_{T} \mathbb{E}(\mathbf{r})$. The result is as follows.

Theorem 2. In both the permittivity and permeability contrast cases, $D_{T} \mathbb{E}(\mathbf{r})$ can be written as

$$
\begin{aligned}
D_{T} \mathbb{E}(\mathbf{r})=\Re \mathfrak{R} \sum_{n=1}^{N}\left(\omega^{2}\left(\varepsilon-\varepsilon_{0}\right)|\mathscr{D}| v^{(n)}(\mathbf{r}) \overline{u^{(n)}(\mathbf{r})}\right. \\
\left.-2|\mathscr{D}| \frac{\mu-\mu_{0}}{\mu+\mu_{0}} \nabla v^{(n)}(\mathbf{r}) \cdot \overline{\nabla u^{(n)}(\mathbf{r})}\right),
\end{aligned}
$$

where $v$ satisfies the following adjoint problem:

$$
\begin{aligned}
& \frac{1}{\mu_{0}} \Delta v^{(n)}(\mathbf{r})+\omega^{2} \varepsilon_{0} v^{(n)}(\mathbf{r})=0 \quad \text { in } \Omega, \\
& \frac{1}{\mu_{0}} \frac{\partial v^{(n)}}{\partial \nu}(\mathbf{r})=u_{\Gamma}^{(n)}(\mathbf{r})-u^{(n)}(\mathbf{r}) \quad \text { on } \partial \Omega .
\end{aligned}
$$

Proof. See the appendix.

Note that, if either $\mu=\mu_{0}$ or $\varepsilon=\varepsilon_{0}$, we can obtain the Fréchet derivatives based on [5, Theorem 3.1] or
[5, Theorem 3.2], respectively. This result is identical to that of [11, Theorem 3.2], but the proof is considerably easier.

\section{Numerical Simulations}

In this section, we choose the background material $\Omega$ as a unit circle centered at origin with $\varepsilon_{0}=\mu_{0}=1$. For thin inclusions $\Gamma_{j}$, the thickness is set to $h=0.02$ and the parameters of are chosen as $\varepsilon_{j}=\mu_{j}=5$, where $\varepsilon_{j}$ and $\mu_{j}$ are the permittivity and permeability contrasts of $\Gamma_{j}$, respectively. The supporting curve $\sigma_{j}$ is selected as

$$
\begin{gathered}
\sigma_{1}=\left\{\left(x, 0.5 x^{2}+0.1\right): x \in[-0.5,0.5]\right\}, \\
\sigma_{2}=\left\{\left(x+0.2, x^{3}+x^{2}-0.6\right): x \in[-0.5,0.5]\right\} .
\end{gathered}
$$

For a given inclusion $\Gamma_{j}$, let $u_{\text {true }}(\mathbf{r})$ be the solution to (3) with boundary condition (4). In the same manner, for an inclusion $\Gamma_{j}^{(s)}$ at the iteration step $s$ (if $s=0$, it is an initial guess), let $u_{\text {comp }}^{(s)}$ denote the solution to (3) with boundary condition (4). For the least-square functional at a given frequency, we introduce a normalized error function:

$$
\mathbb{R}(s)=\frac{\left\|u_{\text {true }}\left(\mathbf{r}_{n}\right)-u_{\text {comp }}^{(s)}\left(\mathbf{r}_{n}\right)\right\|_{\ell^{2}}^{2}}{\left\|u_{\text {comp }}^{(s)}\left(\mathbf{r}_{n}\right)\right\|_{\ell^{2}}^{2}} \text { for } \mathbf{r}_{n} \in \partial \Omega,
$$

where

$$
\left\|f\left(\mathbf{r}_{n}\right)\right\|_{\ell^{2}}^{2}:=\sum_{n=1}^{N}\left|f\left(\mathbf{r}_{n}\right)\right|^{2} .
$$

In this section, we adopt $N=16$ and the locations $\mathbf{r}_{n} \in \partial \Omega$ are selected as

$$
\mathbf{r}_{n}=\left(\cos \frac{2 n \pi}{N}, \sin \frac{2 n \pi}{N}\right), \quad n=1,2, \ldots, N .
$$

A finite-difference time discretization form of (10) is

$$
\phi^{(s+1)}(\mathbf{r})=\phi^{(s)}(\mathbf{r})+\Delta t(s) \mathbb{F}^{(s)}(\mathbf{r})\left|\nabla \phi^{(s)}(\mathbf{r})\right| .
$$

For the evolving level sets $\varphi$ and $\psi$, the time step is chosen as $\Delta t(s)=3 \times 10^{-s}$ (see $[5,12]$ for a detailed discussion). With this configuration, the forward and adjoint problems are solved via the finite element method (FEM) to avoid an inverse crime, and a $20 \mathrm{~dB}$ white Gaussian random noise is added to the boundary measurements data in order to present the robustness.

Remark 3 (generating a good initial guess). It is worth mentioning that, for a successful performance of the iterative based algorithm, one must start the iteration procedure with a good initial guess that is close to the unknown target. Without this guess, one will encounter unexpected scenarios such as nonconvergence (refer to Figures 7 and 14 in [5]). There are various noniterative shape reconstruction algorithms such as the multiple signal classification (MUSIC)-type algorithm [13-17], the end-point identification algorithm [18-20], the multifrequency based subspace migration imaging technique 


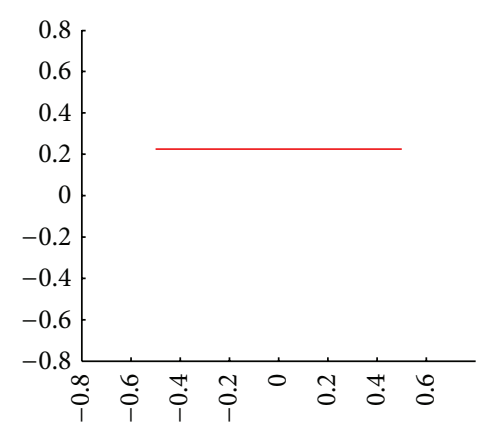

(a) Initial guess

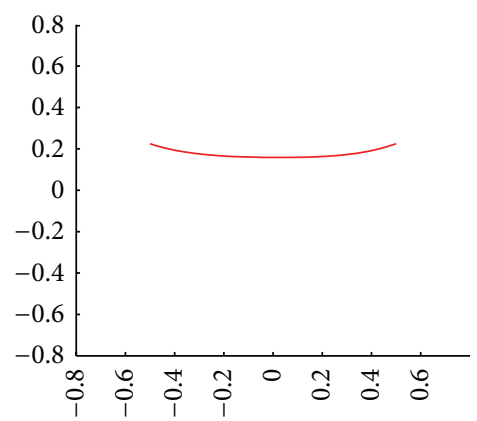

(d) Shape of $\Gamma_{1}^{(100)}$

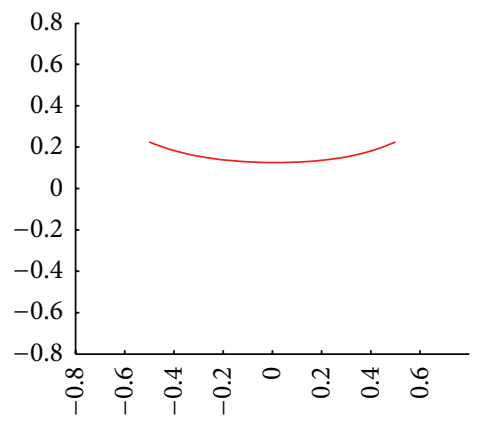

(g) Shape of $\Gamma_{1}^{(200)}$

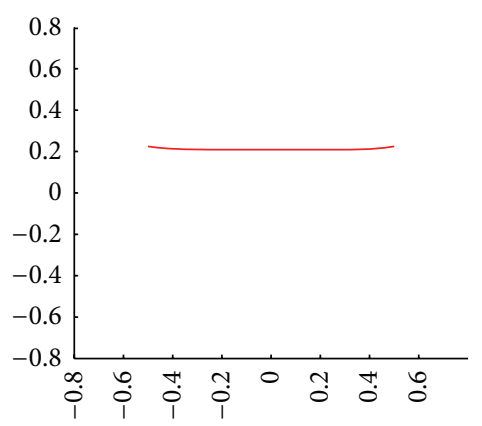

(b) Shape of $\Gamma_{1}^{(20)}$

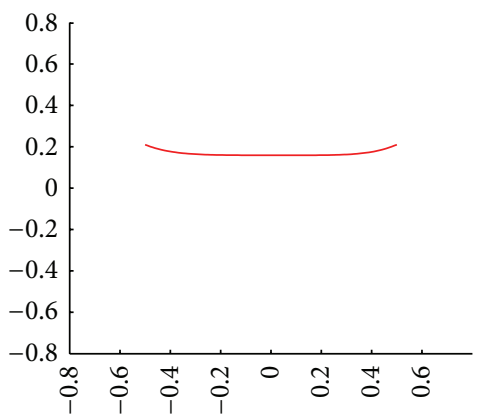

(e) Shape of $\Gamma_{1}^{(140)}$

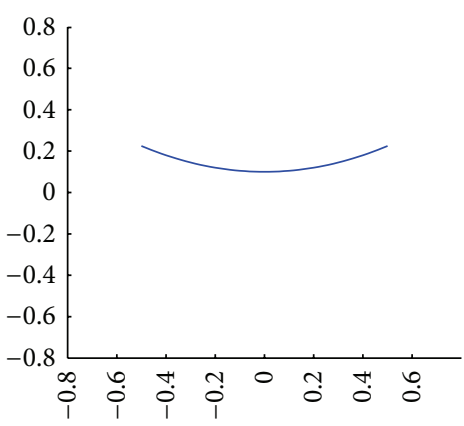

(h) Shape of $\Gamma_{1}$

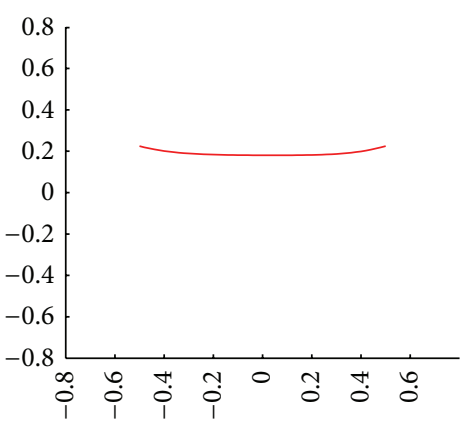

(c) Shape of $\Gamma_{1}^{(60)}$

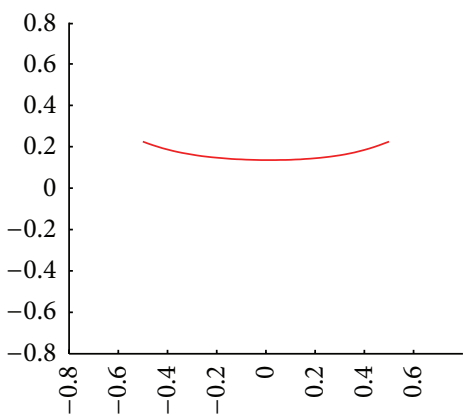

(f) Shape of $\Gamma_{1}^{(160)}$

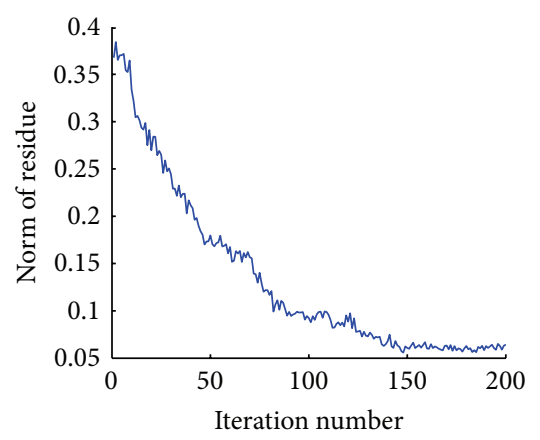

(i) Value of $\mathbb{R}(s)$

FIgURE 2: Reconstruction of $\Gamma_{1}$ with noisy data.

[21-27], the topological derivative strategy [10, 28-32], and the gradient-for-the-initiation (GFI) method [33].

First, we consider the shape reconstruction of $\Gamma_{1}$. In this example, we assume that the location of the end points of $\Gamma_{1}$ has been identified based on the location search algorithm in [18-20]. Thus, by connecting them by a straight line segment, a good initial guess can be obtained.

Figure 2 shows the shape reconstruction of $\Gamma_{1}$ at the operated frequency $\omega=2 \pi / 0.6$. Based on this result, we can confirm that the shape of $\Gamma_{1}$ is successfully reconstructed and the residual considerably decreases accordingly.

Next, we consider the reconstruction of $\Gamma_{2}$. In contrast to the previous example, we apply the topological derivative strategy introduced in $[10,30]$ to obtain a good initial guess. In Figure 3, the obtained shape of $\Gamma_{2}$ via the topological derivative with $N=16$ and $\omega=2 \pi / 0.4$ is illustrated. In this result, we can construct a supporting curve via well-identified points as

$$
\begin{aligned}
\sigma_{2}^{(0)}= & -0.4308 T_{0}(x)+0.4160 T_{1}(x) \\
& +0.1482 T_{2}(x)+0.1895 T_{3}(x) \\
= & 0.7580 x^{3}+0.2964 x^{2}-0.1525 x-0.5790
\end{aligned}
$$

for $x \in[-0.2451,0.6646]$ (see Figure 9 in [10]). Here, $T_{j}(x)$ denotes the Chebyshev polynomials of the first kind. Considering this curve as the supporting curve of the initial guess, the reconstruction of the complete shape of $\Gamma_{2}$ is illustrated in Figure 3. Note that, because of the considerably close initial guess, only 31 iterations are sufficient to obtain a good result. 


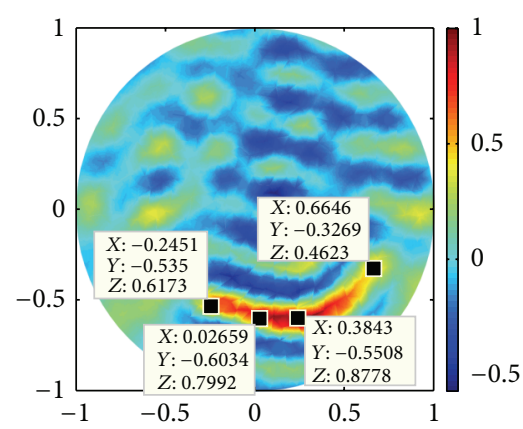

(a) Identified points

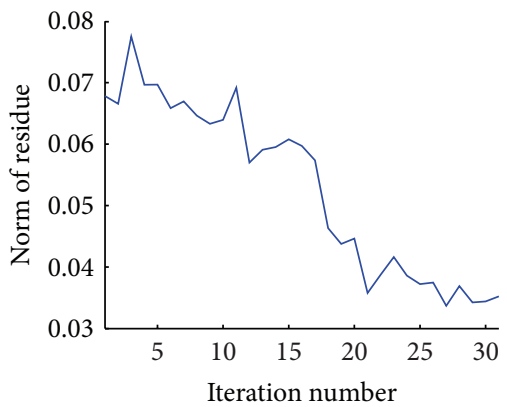

(d) Value of $\mathbb{R}(s)$

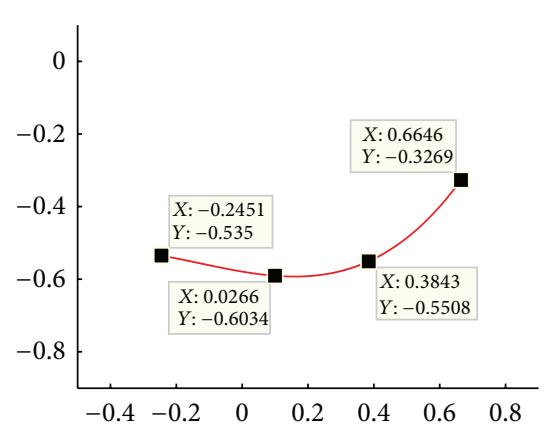

(b) Initial guess

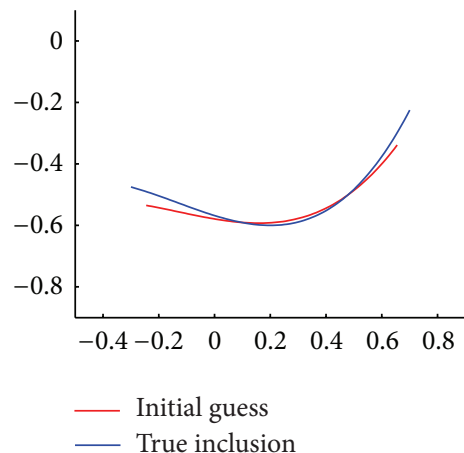

(e) Shape of $\Gamma_{2}^{(0)}$ and $\Gamma_{2}$

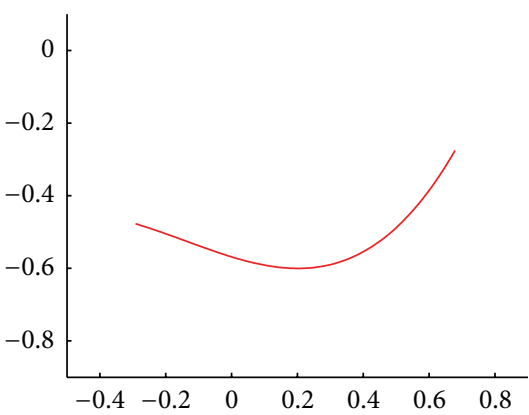

(c) 31 iterations

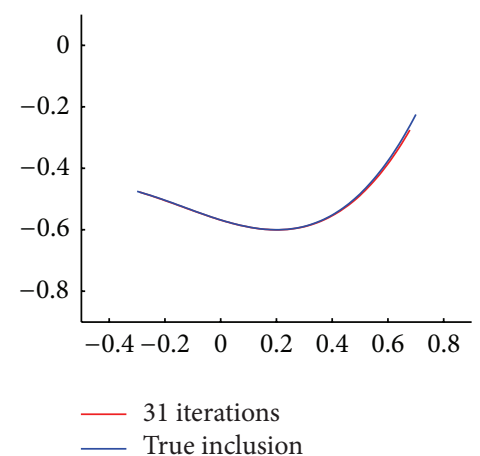

(f) Shape of $\Gamma_{2}^{(31)}$ and $\Gamma_{2}$

Figure 3: Reconstruction of $\Gamma_{2}$ with noisy data.

\section{Conclusion Remarks}

In this paper, a level-set method to perform the shape reconstruction of thin electromagnetic inclusion using both the dielectric permittivity and magnetic permeability contrasts with respect to the homogeneous domain was considered. The relationship between the topological derivative and the evolution speed of level-set functions allowed us to reconstruct the complete shape of the thin inclusion with a good initial guess. It is discovered from the numerical experiments that the proposed technique is stable with respect to random noise. Further, incorporating the topological derivative guarantees a successful reconstruction procedure.

In this paper, we performed shape reconstruction with a priori information of unknown targets, for example, thickness, permittivity, and permeability values. Performing simultaneous reconstruction without a priori information will be a challenge (refer to [3, Section 10.3]).

\section{Appendix}

Proof of Theorem 2. In this appendix, we prove Theorem 2. In order to derive $D_{T} \mathbb{E}(\mathbf{r})$ of (13), we construct a small electromagnetic inclusion $\Sigma=\mathbf{r}+\rho \mathscr{D}$ in $\Omega$ with the same permittivity and permeability of $\Gamma$ and denote this domain by $\Omega \vee \Sigma$. Then, by virtue in [10], the following asymptotic expansion holds:

$$
\mathbb{E}(\Omega \vee \Sigma)=\mathbb{E}(\Omega)+f(\rho) D_{T} \mathbb{E}(\mathbf{r})+o(f(\rho)),
$$

where $f(\rho) \rightarrow 0$ as $\rho \rightarrow 0+$. Let $u_{\Sigma}^{(n)}(\mathbf{r})$ be a solution to (3) with boundary condition (4) in the existence of $\Sigma$. Then, the following asymptotic expansion formula holds (see [13]):

$$
\begin{aligned}
u_{\Sigma}^{(n)}\left(\mathbf{r}^{\prime}\right)- & u^{(n)}\left(\mathbf{r}^{\prime}\right) \\
=\rho^{2}[ & \frac{\mu-\mu_{0}}{\mu+\mu_{0}}|\mathscr{D}| \nabla u^{(n)}(\mathbf{r}) \cdot \nabla \mathcal{N}\left(\mathbf{r}, \mathbf{r}^{\prime}\right) \\
& \left.+\omega^{2}\left(\varepsilon-\varepsilon_{0}\right)|\mathscr{D}| u^{(n)}(\mathbf{r}) \mathcal{N}\left(\mathbf{r}, \mathbf{r}^{\prime}\right)\right]+o\left(\rho^{2}\right),
\end{aligned}
$$

where $\mathcal{N}\left(\mathbf{r}, \mathbf{r}^{\prime}\right)$ denotes the Neumann function that is the solution to

$$
\begin{gathered}
\frac{1}{\mu_{0}} \Delta \mathcal{N}\left(\mathbf{r}, \mathbf{r}^{\prime}\right)+\omega^{2} \varepsilon_{0} \mathcal{N}\left(\mathbf{r}, \mathbf{r}^{\prime}\right)=-\delta\left(\mathbf{r}, \mathbf{r}^{\prime}\right), \quad \mathbf{r} \in \Omega, \\
\frac{1}{\mu_{0}} \frac{\partial \mathcal{N}\left(\mathbf{r}, \mathbf{r}^{\prime}\right)}{\partial \nu(\mathbf{r})}=0, \quad \mathbf{r} \in \partial \Omega .
\end{gathered}
$$

Then, applying (A.2) to (13), we can compute

$$
\begin{aligned}
\mathbb{E}(\Omega \vee \Sigma) & =\frac{1}{2} \sum_{n=1}^{N}\left\|u_{\Gamma}^{(n)}\left(\mathbf{r}^{\prime}\right)-u_{\Sigma}^{(n)}\left(\mathbf{r}^{\prime}\right)\right\|_{L^{2}(\partial \Omega)}^{2} \\
& =\mathbb{E}(\Omega)+\sum_{n=1}^{N} \mathbb{E}_{\Sigma}(\mathbf{r})+o\left(\rho^{2}\right),
\end{aligned}
$$


where

$$
\begin{aligned}
\mathbb{E}_{\Sigma}(\mathbf{r})=\int_{\partial \Omega} & \left(u_{\Gamma}^{(n)}\left(\mathbf{r}^{\prime}\right)-u^{(n)}\left(\mathbf{r}^{\prime}\right)\right) \\
& \times\left(\overline{u^{(n)}\left(\mathbf{r}^{\prime}\right)-u_{\Sigma}^{(n)}\left(\mathbf{r}^{\prime}\right)}\right) d S\left(\mathbf{r}^{\prime}\right) .
\end{aligned}
$$

Then, applying boundary condition (16) and asymptotic formula (A.2) yields

$$
\begin{aligned}
& \mathbb{E}_{\Sigma}(\mathbf{r}) \int_{\partial \Omega}\left[\frac{1}{\mu_{0}} \frac{\partial v^{(n)}}{\partial \nu}\left(\mathbf{r}^{\prime}\right)\right. \\
& \times\left(\rho^{2} \frac{\mu-\mu_{0}}{\mu+\mu_{0}}|\mathscr{D}| \nabla u^{(n)}(\mathbf{r}) \cdot \nabla \mathcal{N}\left(\mathbf{r}, \mathbf{r}^{\prime}\right)\right. \\
&\left.\left.+\rho^{2} \omega^{2}\left(\varepsilon-\varepsilon_{0}\right)|\mathscr{D}| u^{(n)}(\mathbf{r}) \mathcal{N}\left(\mathbf{r}, \mathbf{r}^{\prime}\right)\right)\right] d S\left(\mathbf{r}^{\prime}\right) \\
&=\left(\rho^{2} \omega^{2}\left(\varepsilon-\varepsilon_{0}\right)|\mathscr{D}|\right) \mathbb{E}_{\varepsilon}(\mathbf{r})+\left(\rho^{2} \frac{\mu-\mu_{0}}{\mu+\mu_{0}}|\mathscr{D}|\right) \mathbb{E}_{\mu}(\mathbf{r}) .
\end{aligned}
$$

First, applying integration by parts and (A.3), we can compute $\mathbb{E}_{\varepsilon}(\mathbf{r})$ as

$$
\begin{aligned}
\mathbb{E}_{\varepsilon}(\mathbf{r})= & \int_{\partial \Omega} \frac{1}{\mu_{0}} \frac{\partial v^{(n)}}{\partial v}\left(\mathbf{r}^{\prime}\right) \overline{u^{(n)}(\mathbf{r}) \mathcal{N}\left(\mathbf{r}, \mathbf{r}^{\prime}\right)} d S\left(\mathbf{r}^{\prime}\right) \\
= & \int_{\Omega}\left(\nabla \cdot \frac{1}{\mu_{0}} \frac{\partial v^{(n)}}{\partial v}\left(\mathbf{r}^{\prime}\right)\right) \overline{u^{(n)}(\mathbf{r}) \mathcal{N}\left(\mathbf{r}, \mathbf{r}^{\prime}\right)} d \mathbf{r}^{\prime} \\
& +\int_{\Omega} \nabla v^{(n)}\left(\mathbf{r}^{\prime}\right) \cdot\left(\overline{u^{(n)}(\mathbf{r}) \frac{1}{\mu_{0}} \nabla \mathcal{N}\left(\mathbf{r}, \mathbf{r}^{\prime}\right)}\right) d \mathbf{r}^{\prime} \\
= & \int_{\Omega}\left(\frac{1}{\mu_{0}} \Delta v^{(n)}\left(\mathbf{r}^{\prime}\right)\right) \overline{u^{(n)}(\mathbf{r}) \mathcal{N}\left(\mathbf{r}, \mathbf{r}^{\prime}\right)} d \mathbf{r}^{\prime} \\
& +\int_{\Omega} \omega^{2} \varepsilon_{0} v^{(n)}\left(\mathbf{r}^{\prime}\right) \overline{u^{(n)}(\mathbf{r}) \mathcal{N}\left(\mathbf{r}, \mathbf{r}^{\prime}\right)} d \mathbf{r}^{\prime} \\
& -\int_{\Omega} v^{(n)}\left(\mathbf{r}^{\prime}\right) \overline{u^{(n)}(\mathbf{r})\left(\frac{1}{\mu_{0}} \Delta \mathcal{N}\left(\mathbf{r}, \mathbf{r}^{\prime}\right)\right)} d \mathbf{r}^{\prime} \\
& +\int_{\Omega} \omega^{2} \varepsilon_{0} v^{(n)}\left(\mathbf{r}^{\prime}\right) \overline{u^{(n)}(\mathbf{r}) \mathcal{N}\left(\mathbf{r}, \mathbf{r}^{\prime}\right)} d \mathbf{r}^{\prime} \\
= & v^{(n)}(\mathbf{r}) \overline{u^{(n)}(\mathbf{r}) .}
\end{aligned}
$$

Similarly, $\mathbb{E}_{\mu}(\mathbf{r})$ becomes

$$
\begin{aligned}
\mathbb{E}_{\mu}(\mathbf{r}) & =\int_{\partial \Omega} \frac{1}{\mu_{0}} \frac{\partial v^{(n)}}{\partial \nu}\left(\mathbf{r}^{\prime}\right) \overline{\nabla u^{(n)}(\mathbf{r}) \cdot \nabla \mathcal{N}\left(\mathbf{r}, \mathbf{r}^{\prime}\right)} d S\left(\mathbf{r}^{\prime}\right) \\
& =\int_{\Omega}\left(\frac{1}{\mu_{0}} \Delta v^{(n)}\left(\mathbf{r}^{\prime}\right)\right) \overline{\nabla u^{(n)}(\mathbf{r}) \nabla \mathcal{N}\left(\mathbf{r}, \mathbf{r}^{\prime}\right)} d \mathbf{r}^{\prime}
\end{aligned}
$$

$$
\begin{aligned}
& +\int_{\Omega} \nabla v^{(n)}\left(\mathbf{r}^{\prime}\right) \cdot\left(\overline{\nabla u^{(n)}(\mathbf{r}) \frac{1}{\mu_{0}} \Delta \mathcal{N}\left(\mathbf{r}, \mathbf{r}^{\prime}\right)}\right) d \mathbf{r}^{\prime} \\
= & -\int_{\Omega} \omega^{2} \varepsilon_{0} v^{(n)}\left(\mathbf{r}^{\prime}\right) \overline{\nabla u^{(n)}(\mathbf{r}) \nabla \mathcal{N}\left(\mathbf{r}, \mathbf{r}^{\prime}\right)} d \mathbf{r}^{\prime} \\
& -\int_{\Omega} v^{(n)}\left(\mathbf{r}^{\prime}\right) \overline{\nabla u^{(n)}(\mathbf{r}) \nabla\left(\frac{1}{\mu_{0}} \Delta \mathcal{N}\left(\mathbf{r}, \mathbf{r}^{\prime}\right)\right)} d \mathbf{r}^{\prime} \\
= & \int_{\Omega}-v^{(n)}\left(\mathbf{r}^{\prime}\right) \overline{\nabla u^{(n)}(\mathbf{r})} \\
& \times \frac{\nabla\left(\frac{1}{\mu_{0}} \Delta \mathcal{N}\left(\mathbf{r}, \mathbf{r}^{\prime}\right)+\omega^{2} \varepsilon_{0} \mathcal{N}\left(\mathbf{r}, \mathbf{r}^{\prime}\right)\right)}{r^{\prime}} \mathbf{r}^{\prime} \\
= & \int_{\Omega} \nabla v^{(n)}\left(\mathbf{r}^{\prime}\right) \cdot \overline{\nabla u^{(n)}(\mathbf{r})} \\
& \times \frac{1}{\mu_{0} \Delta \mathcal{N}\left(\mathbf{r}, \mathbf{r}^{\prime}\right)+\omega^{2} \varepsilon_{0} \mathcal{N}\left(\mathbf{r}, \mathbf{r}^{\prime}\right)} d \mathbf{r}^{\prime} \\
= & -\nabla v^{(n)}(\mathbf{r}) \overline{\nabla u^{(n)}(\mathbf{r})} .
\end{aligned}
$$

Hence,

$$
\begin{aligned}
\mathbb{E}_{\Sigma}(\mathbf{r})=\rho^{2}( & \omega^{2}\left(\varepsilon-\varepsilon_{0}\right) v^{(n)}(\mathbf{r}) \overline{u^{(n)}(\mathbf{r})} \\
& \left.-2|\mathscr{D}| \frac{\mu-\mu_{0}}{\mu+\mu_{0}} \nabla v^{(n)}(\mathbf{r}) \cdot \overline{\nabla u^{(n)}(\mathbf{r})}\right),
\end{aligned}
$$

and by considering the real part (A.9) and comparing (A.1) and (A.4), we can observe that $f(\rho)=\rho^{2}$ and

$$
\begin{aligned}
D_{T} \mathbb{E}(\mathbf{r})=\mathfrak{R e} \sum_{n=1}^{N}( & \omega^{2}\left(\varepsilon-\varepsilon_{0}\right)|\mathscr{D}| v^{(n)}(\mathbf{r}) \overline{u^{(n)}(\mathbf{r})} \\
& \left.-2|\mathscr{D}| \frac{\mu-\mu_{0}}{\mu+\mu_{0}} \nabla v^{(n)}(\mathbf{r}) \cdot \overline{\nabla u^{(n)}(\mathbf{r})}\right) .
\end{aligned}
$$

This completes the proof.

\section{Acknowledgments}

The author would like to acknowledge the anonymous reviewer for his valuable comments. This work was supported by the Basic Science Research Program through the National Research Foundation of Korea (NRF), funded by the Ministry of Education, Science, and Technology (no. 2011-0007705), and the Research Program of the Kookmin University in Korea.

\section{References}

[1] H. Ammari, An Introduction to Mathematics of Emerging Biomedical Imaging, vol. 62 of Mathematics and Applications, Springer, Berlin, Germany, 2008. 
[2] F. Santosa, "A level-set approach for inverse problems involving obstacles," ESAIM: Control, Optimisation and Calculus of Variations, vol. 1, pp. 17-33, 1996.

[3] O. Dorn and D. Lesselier, "Level set methods for inverse scattering," Inverse Problems, vol. 22, no. 4, pp. R67-R131, 2006.

[4] D. Álvarez, O. Dorn, and M. Moscoso, "Reconstructing thin shapes from boundary electrical measurements with level sets," International Journal of Information \& Systems Sciences, vol. 2, no. 4, pp. 498-511, 2006.

[5] W. K. Park and D. Lesselier, "Reconstruction of thin electromagnetic inclusions by a level-set method," Inverse Problems, vol. 25, no. 8, Article ID 085010, 2009.

[6] M. Burger, B. Hackl, and W. Ring, "Incorporating topological derivatives into level set methods," Journal of Computational Physics, vol. 194, no. 1, pp. 344-362, 2004.

[7] I. Cimrák, "Material and shape derivative method for quasilinear elliptic systems with applications in inverse electromagnetic interface problems," SIAM Journal on Numerical Analysis, vol. 50, no. 3, pp. 1086-1110, 2012.

[8] G. R. Feijoo, "A new method in inverse scattering based on the topological derivative," Inverse Problems, vol. 20, no. 6, pp. 1819$1840,2004$.

[9] L. He, C. Y. Kao, and S. Osher, "Incorporating topological derivatives into shape derivatives based level set methods," Journal of Computational Physics, vol. 225, no. 1, pp. 891-909, 2007.

[10] W. K. Park, “Topological derivative strategy for one-step iteration imaging of arbitrary shaped thin, curve-like electromagnetic inclusions," Journal of Computational Physics, vol. 231, no. 4, pp. 1426-1439, 2012.

[11] A. Carpio and M. L. Rapún, "Solving inhomogeneous inverse problems by topological derivative methods," Inverse Problems, vol. 24, no. 4, Article ID 045014, 2008.

[12] C. Ramananjaona, M. Lambert, D. Lesselier, and J. P. Zolésio, "Shape reconstruction of buried obstacles by controlled evolution of a level set: from a min-max formulation to numerical experimentation," Inverse Problems, vol. 17, no. 4, pp. 1087-1111, 2001.

[13] H. Ammari and H. Kang, Polarization and Moment Tensors: With Applications to Inverse Problems, and Effective Medium Theory, vol. 162 of Applied Mathematical Sciences, Springer, New York, NY, USA, 2007.

[14] H. Ammari, H. Kang, H. Lee, and W. K. Park, "Asymptotic imaging of perfectly conducting cracks," SIAM Journal on Scientific Computing, vol. 32, no. 2, pp. 894-922, 2010.

[15] Y. D. Joh and W. K. Park, "Structural behavior of the MUSICtype algorithm for imaging perfectly conducting cracks," Progress in Electromagnetics Research, vol. 138, pp. 211-226, 2013.

[16] W. K. Park and D. Lesselier, "Electromagnetic MUSIC-type imaging of perfectly conducting, arc-like cracks at single frequency," Journal of Computational Physics, vol. 228, no. 21, pp. 8093-8111, 2009.

[17] W. K. Park and D. Lesselier, "MUSIC-type imaging of a thin penetrable inclusion from its multi-static response matrix," Inverse Problems, vol. 25, no. 7, Article ID 075002, 2009.

[18] H. Ammari, E. Beretta, and E. Francini, "Reconstruction of thin conductivity imperfections," Applicable Analysis, vol. 83, no. 1, pp. 63-76, 2004.

[19] H. Ammari, E. Beretta, and E. Francini, "Reconstruction of thin conductivity imperfections. II. The case of multiple segments," Applicable Analysis, vol. 85, no. 1-3, pp. 87-105, 2006.
[20] H. Lee and W. K. Park, "Location search algorithm of thin conductivity inclusions via boundary measurements," ESAIM: Proceedings, vol. 26, pp. 217-229, 2009.

[21] H. Ammari, J. Garnier, H. Kang, W. K. Park, and K. Sølna, "Imaging schemes for perfectly conducting cracks," SIAM Journal on Applied Mathematics, vol. 71, no. 1, pp. 68-91, 2011.

[22] Y. D. Joh, Y. M. Kwon, J. Y. Huh, and W. K. Park, "Structure analysis of single- and multi-frequency subspace migrations in inverse scattering problems," Progress in Electromagnetics Research, vol. 136, pp. 607-622, 2013.

[23] Y. M. Kwon and W. K. Park, "Analysis of subspace migrations in limited-view inverse scattering problems," Applied Mathematics Letters, vol. 26, no. 12, pp. 1107-1113, 2013.

[24] W. K. Park, "Non-iterative imaging of thin electromagnetic inclusions from multi-frequency response matrix," Progress in Electromagnetics Research, vol. 106, pp. 225-241, 2010.

[25] W. K. Park, "On the imaging of thin dielectric inclusions buried within a half-space," Inverse Problems, vol. 26, no. 7, Article ID 074008, 2010.

[26] W. K. Park and D. Lesselier, "Fast electromagnetic imaging of thin inclusions in half-space affected by random scatterers," Waves in Random and Complex Media, vol. 22, no. 1, pp. 3-23, 2012.

[27] W. K. Park and T. Park, "Multi-frequency based location search algorithm of small electromagnetic inhomogeneties embedded in two-layered medium," Computer Physics Communications, vol. 184, no. 7, pp. 1649-1659, 2013.

[28] Y. K. Ma, P. S. Kim, and W. K. Park, "Analysis of topological derivative function for a fast electromagnetic imaging of perfectly conducing cracks," Progress in Electromagnetics Research, vol. 122, pp. 311-325, 2011.

[29] Y. K. Ma and W. K. Park, "A topological derivative based non-iterative electromagnetic imaging of perfectly conducting cracks," Journal of Electromagnetic Engineering and Science, vol. 12, no. 1, pp. 128-134, 2012.

[30] W. K. Park, "Multi-frequency topological derivative for approximate shape acquisition of curve-like thin electromagnetic inhomogeneities," Journal of Mathematical Analysis and Applications, vol. 404, no. 2, pp. 501-518, 2013.

[31] W. K. Park, "On the imaging of thin dielectric inclusions via topological derivative concept," Progress in Electromagnetics Research, vol. 110, pp. 237-252, 2010.

[32] W. K. Park, “Topological derivative for fast imaging of twodimensional thin dielectric inclusions in the wave propagation environment," Journal of Electromagnetic Engineering and Science, vol. 11, no. 1, pp. 56-61, 2011.

[33] I. Cimrák and R. van Keer, "Level set method for the inverse elliptic problem in nonlinear electromagnetism," Journal of Computational Physics, vol. 229, no. 24, pp. 9269-9283, 2010. 


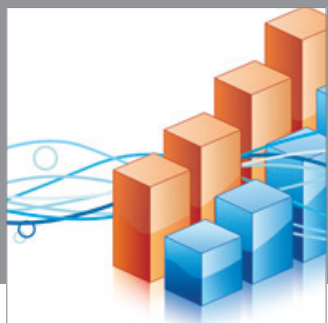

Advances in

Operations Research

mansans

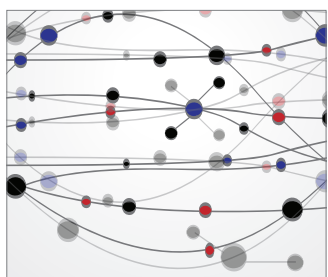

The Scientific World Journal
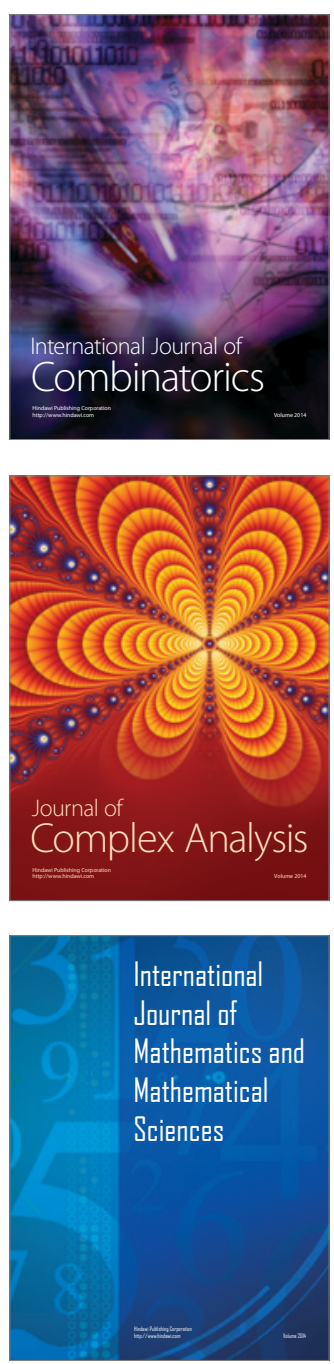
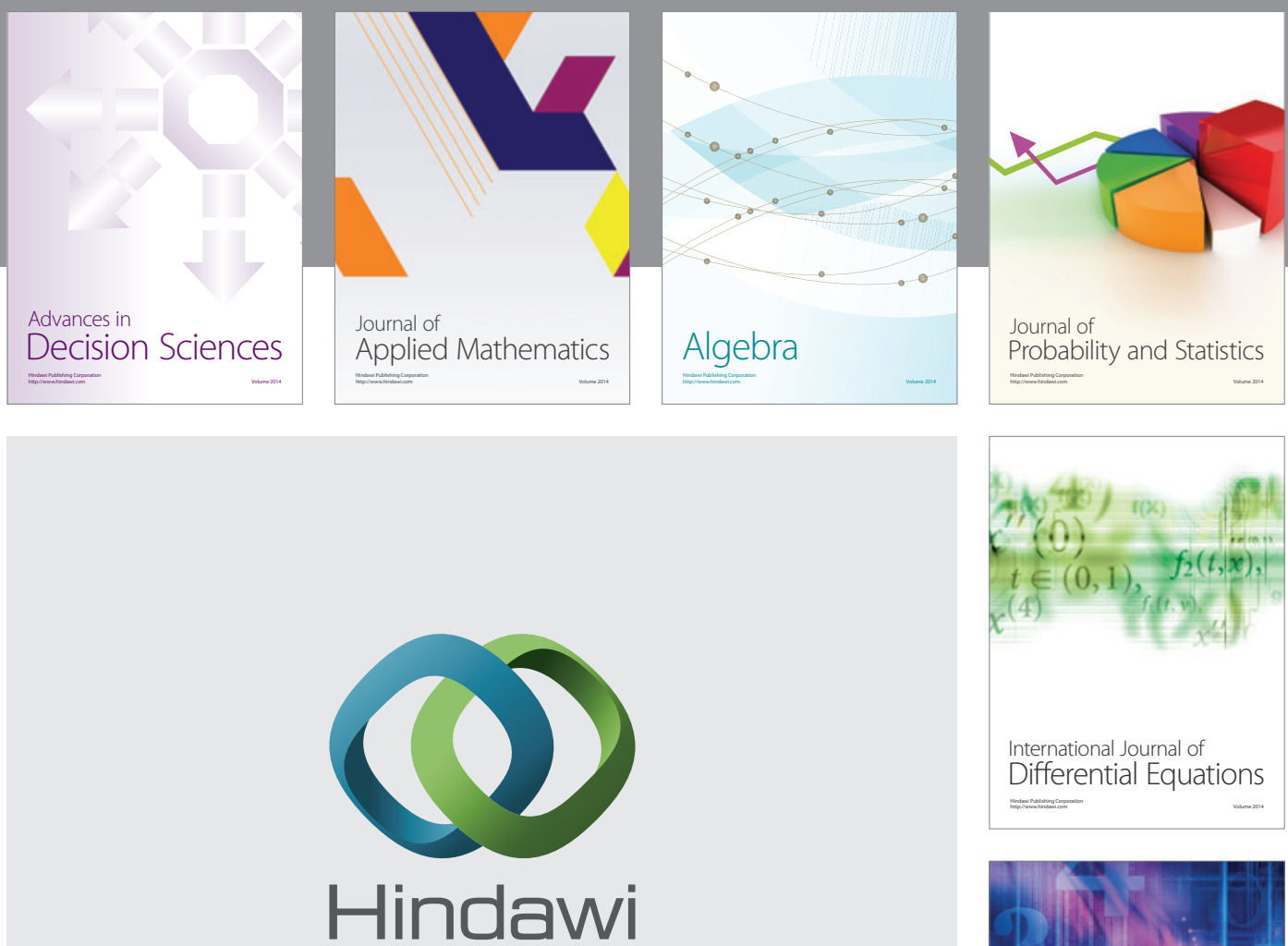

Submit your manuscripts at http://www.hindawi.com
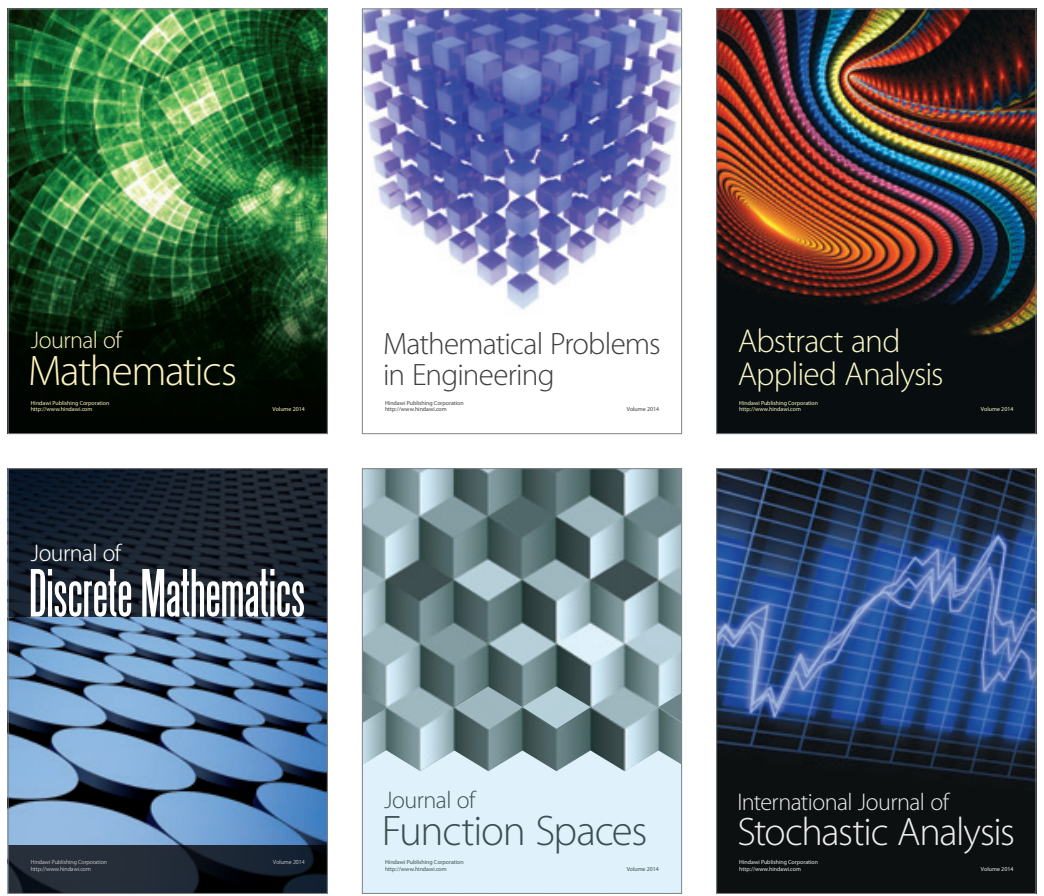

Journal of

Function Spaces

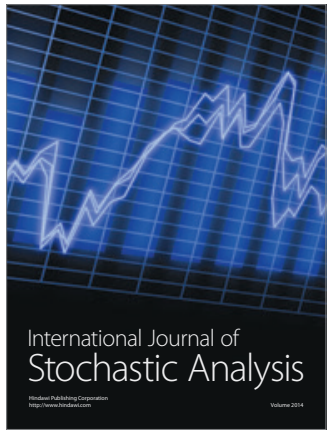

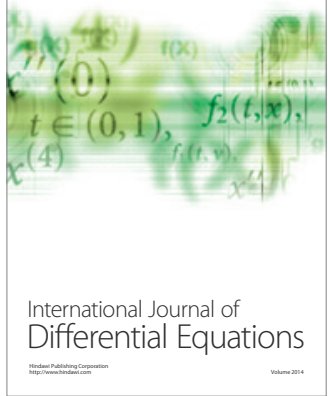
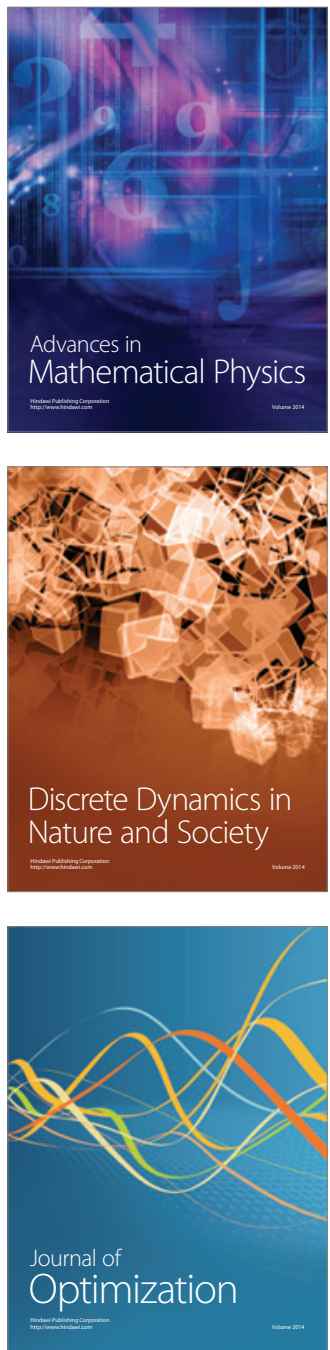